\title{
High Gamma Power Is Phase-Locked to Theta Oscillations in Human Neocortex
}

\author{
R. T. Canolty ${ }^{1,}{ }^{*}$, E. Edwards ${ }^{1,2}$, S. S. Dalal ${ }^{3}$, M. Soltani ${ }^{1,2}$, S. S. Nagarajan ${ }^{3,4}$, H. E. \\ Kirsch $^{5}$, M. S. Berger 6 , N. M. Barbaro ${ }^{5,6}$, and R. T. Knight ${ }^{1,2,3,5,6}$ \\ 1 Helen Wills Neuroscience Institute, University of California, Berkeley, CA 94720, USA \\ 2Department of Psychology, University of California, Berkeley, CA 94720, USA \\ 3Department of Bioengineering, University of California, San Francisco, CA 94143, USA \\ 4Department of Radiology, University of California, San Francisco, CA 94143, USA \\ 5Department of Neurology, University of California, San Francisco, CA 94143, USA \\ 6Department of Neurosurgery, University of California, San Francisco, CA 94143, USA
}

\section{Abstract}

We observed robust coupling between the high- and low-frequency bands of ongoing electrical activity in the human brain. In particular, the phase of the low-frequency theta (4 to 8 hertz) rhythm modulates power in the high gamma ( 80 to 150 hertz) band of the electrocorticogram, with stronger modulation occurring at higher theta amplitudes. Furthermore, different behavioral tasks evoke distinct patterns of theta/high gamma coupling across the cortex. The results indicate that transient coupling between low- and high-frequency brain rhythms coordinates activity in distributed cortical areas, providing a mechanism for effective communication during cognitive processing in humans.

Neuronal oscillations facilitate synaptic plasticity (1), influence reaction time (2), correlate with attention (3) and perceptual binding (4), and are proposed to play a role in transient, longrange coordination of distinct brain regions (5). Direct cortical recordings reveal that ongoing rhythms encompass a wide range of spatial and temporal scales - ultraslow rhythms less than $0.05 \mathrm{~Hz}$ coexist with fast transient oscillations $500 \mathrm{~Hz}$ or greater (1), with spatial coherence between these oscillations extending from several centimeters for the corticospinal tract (6) to the micrometer scale for subthreshold membrane oscillations in a single neuron (7). Exactly how these transient oscillations influence each other and coordinate processing at both the single-neuron and population levels remains unknown.

Evidence for cross-frequency coupling, where one frequency band modulates the activity of a different frequency band, is more abundant in animal than human data. For example, the theta rhythm can modulate the firing rate and spike timing of a single neuron (8-11) as well as the gamma power of the intracortical local field potential $(8,12,13)$. Task-related changes in theta power have been observed in humans (14-16), and cross-frequency coupling at frequencies up to $40 \mathrm{~Hz}$ has been detected at the scalp $(17,18)$. However, given the difficulty in localizing electrical sources from scalp recordings alone (19), subdural electrodes that record directly from the human cortex are needed to address this question. Furthermore, subdural electrodes are ideal for studying activity in the recently described human high gamma band (HG) at 80 to $150 \mathrm{~Hz}$. HG activity is modulated by sensory, motor, and cognitive events (20), is

\footnotetext{
*To whom correspondence should be addressed. E-mail: rcanolty@berkeley.edu
} 
functionally distinct from low gamma (30 to $80 \mathrm{~Hz}$ ) with different physiological origins (21), and is correlated with the functional magnetic resonance imaging blood oxygen leveldependent (fMRI BOLD) signal (22-24). There have been no reports of coupling between any low-frequency rhythm and HG in signals recorded either at the scalp or directly from human sensory, motor, or association cortex. We therefore focus exclusively on theta/HG coupling in this report.

We analyzed multichannel subdural electrocorticogram (ECoG) data from five patients undergoing neurosurgical treatment for epilepsy. Typically, the events of interest in behavioral paradigms are the stimulus onsets and motor responses that evoke frequency-specific changes in the electrical activity of the brain. In contrast, the events of interest in cross-frequency coupling are features of the ongoing oscillatory activity itself. That is, cross-frequency coupling refers to statistical dependence between distinct frequency bands of the ongoing ECoG rather than dependence between the ECoG and external stimulus events. The dependence between two frequencies $f_{1}$ and $f_{2}$ can assume many forms, including coupling between the amplitude envelopes $A_{1}(t)$ and $A_{2}(t)$, the phase time series $\varphi_{1}(t)$ and $\varphi_{2}(t)$, or an amplitude-phase coupling between $A_{1}(t)$ and $\varphi_{2}(t)$. We focus here on the last type of coupling and use an index of crossfrequency coupling that directly combines the amplitude envelope time series $A_{1}(t+\tau)$ of a high-frequency band with the phase time series $\varphi_{2}(t)$ of alow-frequency band into one composite, complex-valued signal $z(t, \tau)$. The (normalized) temporal mean of this composite signal provides a sensitive measure of the coupling strength and preferred phase between the two frequencies (25).

Animal evidence for theta phase modulation of single-unit firing and the strong connection of theta to learning, attention, and memory $(26,27)$ suggested to us that high-frequency oscillations in human neocortex may be modulated by the theta rhythm. Accordingly, we analyzed the ECoG across a range of behavioral tasks (25). Figure 1B shows a time-frequency plot for data recorded from an electrode over the left middle frontal gyrus during an auditory language-related target detection task (Fig. 1A, arrow). Theta trough-locked averaging of the normalized time-frequency plane shows significant coupling $(P<0.001$, corrected) between theta phase and high-frequency power, with an increase or decrease in power relative to baseline occurring at the theta trough or peak. Theta coupling was broadband from $\sim 20$ to 200 $\mathrm{Hz}$, with the strongest modulation occurring in the HG band. Fig. 1D and fig. S4, using the modulation index discussed above, also show that coupling is strongest between theta phase and $\mathrm{HG}$ amplitude.

Across all tasks and subjects, 252 out of 299 tested electrodes (84.3\%) showed significant theta/ gamma coupling $(P<0.001$ for each electrode, corrected). Excluding the 60 electrodes over resected tissue (which includes both epileptic and healthy tissue) increases this percentage to $88.7 \%$, whereas only $66.7 \%$ of electrodes over resected tissue showed significant coupling. The largest HG amplitudes tended to occur at the trough of the theta waveform in electrodes with strong coupling (Figs. 1C and 2A). The coupling strength between the HG analytic amplitude time series $A_{\mathrm{HG}}(t+\tau)$ and theta analytic phase $\varphi_{\mathrm{TH}}(t)$ should decrease to chance levels as the magnitude of the time lag $t$ increases. Figure $3 \mathrm{~A}$, displaying all $\mathrm{ECoG}$ electrodes for one subject, shows that this is indeed the case (see fig. S8 for all subjects).

The strength of theta/HG coupling depends on theta power as well as theta phase. We observed stronger coupling in electrodes with greater mean theta amplitude (Fig. 2B). That is, HG amplitudes have a stronger theta phase preference at greater theta power, indicating that theta/ HG coupling strength can be modulated by adjusting theta power in a local cortical region. This contrasts with the weak negative correlation observed between theta/HG coupling strength and mean HG amplitude (Fig. 2C and fig. S6). Thus, mean HG power and the strength of theta/ HG coupling appear to reflect independent dimensions of cortical activity. 
Task-dependent modulation of theta power has been shown in humans (26), prompting the hypothesis that theta/HG coupling may be task-dependent. Two examples of task-specific changes in the spatial pattern of theta/HG coupling strength over all electrodes in one subject are shown in Fig. 3, B and C. Figure 3D shows that behavioral tasks evoke distinct and reproducible patterns of coupling in this subject, with similar tasks evoking similar coupling patterns whereas different tasks evoked alternate patterns. Spatial patterns associated with two runs of similar tasks were positively correlated, whereas runs of different tasks exhibited a null or negative correlation. This trend held across all tasks and subjects, as shown by Fig. 3E. These results are consistent with the hypothesis that transient cross-frequency coupling modulates network engagement, enabling flexible control of cognitive processing.

Oscillations are rhythmic fluctuations in neuronal excitability that modulate both output spike timing and sensitivity to synaptic input (5). Therefore, effective communication between neuronal populations requires precise matching of the relative phase of distinct rhythms to axonal conduction delays. An oscillatory hierarchy operating across multiple spatial and temporal scales could regulate this proposed long-range communication (13). Basal forebrain cortical-projecting GABAergic ( $\gamma$-aminobutyric acid-releasing) neurons are well positioned to control theta/HG coupling; these neurons preferentially synapse onto intracortical GABAergic neurons throughout the cortex, with disinhibitory spike bursts causing a brief increase in gamma power at the theta trough (28). Our observations that (i) HG power is modulated by theta phase, (ii) an increase in theta power strengthens theta/HG coupling, and (iii) the topography of theta/HG coupling is task-dependent support the hypothesis that crossfrequency coupling between distinct brain rhythms facilitates the transient coordination of cortical areas required for adaptive behavior in humans.

\section{Supplementary Material}

Refer to Web version on PubMed Central for supplementary material.

\section{References and Notes}

1. Buzsaki G, Draguhn A. Science 2004;304:1926. [PubMed: 15218136]

2. Linkenkaer-Hansen K, Nikulin VV, Palva S, Ilmoniemi RJ, Palva JM. J. Neurosci 2004;24:10186. [PubMed: 15537890]

3. Womelsdorf T, Fries P, Mitra PP, Desimone R. Nature 2006;439:733. [PubMed: 16372022]

4. Lachaux JP, et al. Neuroimage 2005;25:491. [PubMed: 15784428]

5. Fries P. Trends Cogn. Sci 2005;9:474. [PubMed: 16150631]

6. Schoffelen JM, Oostenveld R, Fries P. Science 2005;308:111. [PubMed: 15802603]

7. Izhikevich EM. Neural Netw 2001;14:883. [PubMed: 11665779]

8. Bragin A, et al. J. Neurosci 1995;15:47. [PubMed: 7823151]

9. Buzsaki G, et al. Neuroscience 2003;116:201. [PubMed: 12535953]

10. Siapas AG, Lubenov EV, Wilson MA. Neuron 2005;46:141. [PubMed: 15820700]

11. Lee H, Simpson GV, Logothetis NK, Rainer G. Neuron 2005;45:147. [PubMed: 15629709]

12. Chrobak JJ, Buzsaki G. J. Neurosci 1998;18:388. [PubMed: 9412515]

13. Lakatos P, et al. J. Neurophysiol 2005;94:1904. [PubMed: 15901760]

14. Ekstrom AD, et al. Hippocampus 2005;15:881. [PubMed: 16114040]

15. Sederberg PB, Kahana MJ, Howard MW, Donner EJ, Madsen JR. J. Neurosci 2003;23:10809. [PubMed: 14645473]

16. Onton J, Delorme A, Makeig S. Neuroimage 2005;27:341. [PubMed: 15927487]

17. Schack B, Vath N, Petsche H, Geissler HG, Moller E. Int. J. Psychophysiol 2002;44:143. [PubMed: 11909647]

18. Palva JM, Palva S, Kaila K. J. Neurosci 2005;25:3962. [PubMed: 15829648] 
19. Nunez, PL.; Srinivasan, RS. Electric Fields of the Brain: The Neurophysics of EEG. Oxford Univ. Press; Oxford: 2005.

20. Crone NE, Miglioretti DL, Gordon B, Lesser RP. Brain 1998;121:2301. [PubMed: 9874481]

21. Edwards E, Soltani M, Deouell LY, Berger MS, Knight RT. J. Neurophysiol 2005;94:4269. [PubMed: 16093343]

22. Logothetis NK, Pauls J, Augath M, Trinath T, Oeltermann A. Nature 2001;412:150. [PubMed: 11449264]

23. Mukamel R, et al. Science 2005;309:951. [PubMed: 16081741]

24. Niessing J, et al. Science 2005;309:948. [PubMed: 16081740]

25. Materials and methods are available as supporting material on Science Online

26. Kahana MJ, Seelig D, Madsen JR. Curr. Opin. Neurobiol 2001;11:739. [PubMed: 11741027]

27. Miller, R. Cortico-Hippocampal Interplay and the Representation of Contexts in the Brain. SpringerVerlag; New York: 1991.

28. Lin SC, Gervasoni D, Nicolelis MAL. J. Neurophysiol. 2006doi: 10.1152/jn.00524.2006

29. This work was supported by the Rauch family, National Institute of Neurological Disorders and Stroke grant NS21135, NSF Fellowship 2004016118, and National Institute on Deafness and Other Communication Disorders grant F31DC006762. The authors thank P. Garcia and D. Filippi of the Department of Neurology, University of California San Francisco, for their help on electrical stimulation mapping and localizing epileptic tissue 

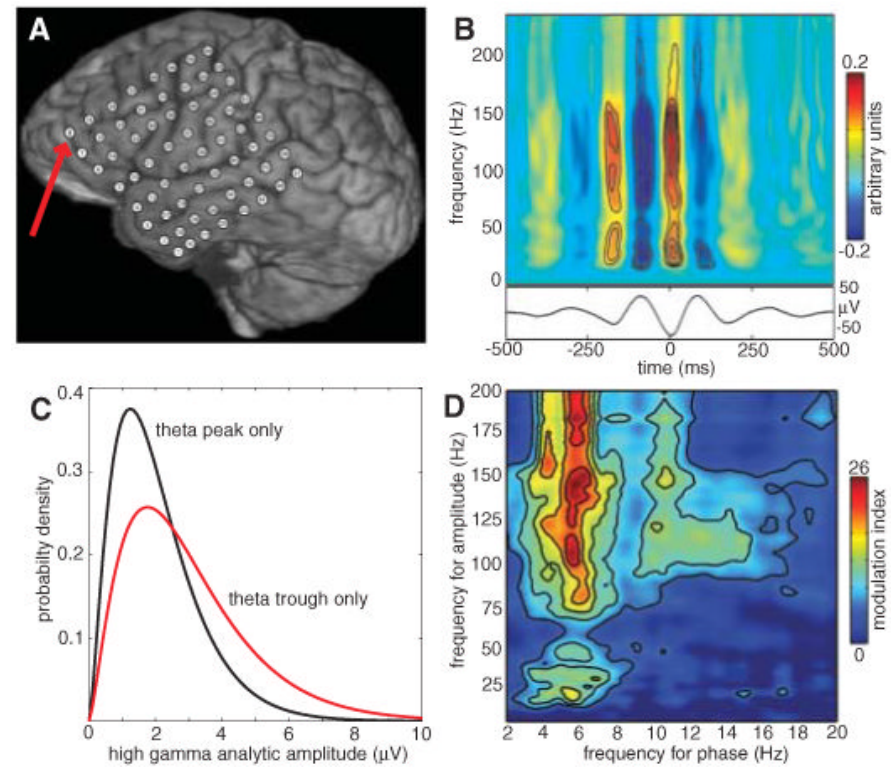

Fig. 1.

High gamma (80 to $150 \mathrm{~Hz}$ ) power is modulated by theta (4 to $8 \mathrm{~Hz}$ ) phase. (A) Structural MRI showing position of 64-channel ECoG grid over frontal and temporal lobes in subject 1. (B) Example of phase-locked modulation of power in the ECoG signal from an electrode over the anterior portion of the middle frontal gyrus (arrow in Fig. 1A). (Top) Time-frequency plot of mean power modulation time-locked to the theta trough. Outermost contour indicates statistical significance $(P<0.001$, corrected). Normalization permits comparison across frequencies; red and blue indicate a power increase or decrease, respectively, relative to the mean power. (Bottom) Theta trough-locked average of raw ECoG signal. (C) Best-fit gamma distributions for the high gamma analytic amplitude values that occurred at the peak (black, 0 radians) or the trough (red, p radians) of the theta waveform for the same electrode as in Fig. 1B. The difference in parameter values is significant $(P<0.001)$. (D) The modulation index $(25)$ as a function of analytic amplitude ( 5 to $200 \mathrm{~Hz}$ ) and analytic phase (2 to $20 \mathrm{~Hz}$ ) for the same electrode as in Fig. 1B. Outermost contour indicates statistical significance $(P<0.001$, corrected). Larger values indicate stronger cross-frequency coupling. Maximal coupling for this electrode is $146.2 \mathrm{~Hz}$ amplitude and $5.6 \mathrm{~Hz}$ phase (see also fig. S4). 

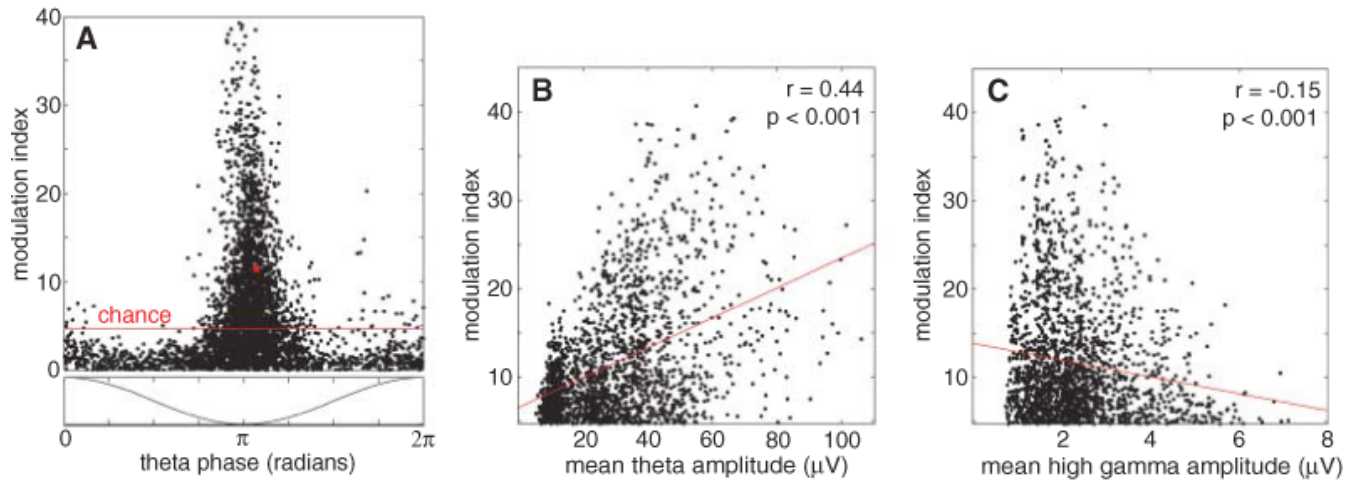

Fig. 2.

Theta/HG coupling strength is a function of theta amplitude. (A) Theta/HG coupling strength and preferred theta phase. (Bottom) One theta cycle (schematic), from theta peak (0 radians) to trough ( $\pi$ radians) to peak ( $\pi$ radians). (Top) Modulation index (25) computed separately for all electrodes in all subjects for each task. Larger magnitudes indicate stronger coupling (vertical axis), whereas the horizontal axis indicates the theta phase at which larger HG amplitudes tend to occur. Most electrodes with strong theta/HG coupling have a preferred theta phase of $\pi$, corresponding to the theta trough (see also Fig. 1C). The red dot indicates the electrode and recording block examined in Fig. 1. The red horizontal line corresponds to the significance threshold after correction for multiple comparisons. (B) Modulation index versus mean theta amplitude for all significant values from Fig. 2A (black dots) and best linear fit (red line), indicating their positive correlation. (C) Modulation index versus mean HG amplitude for all significant values from Fig. 2A (black dots) and best linear fit (red line), indicating their weak negative correlation (see also fig. S6). 

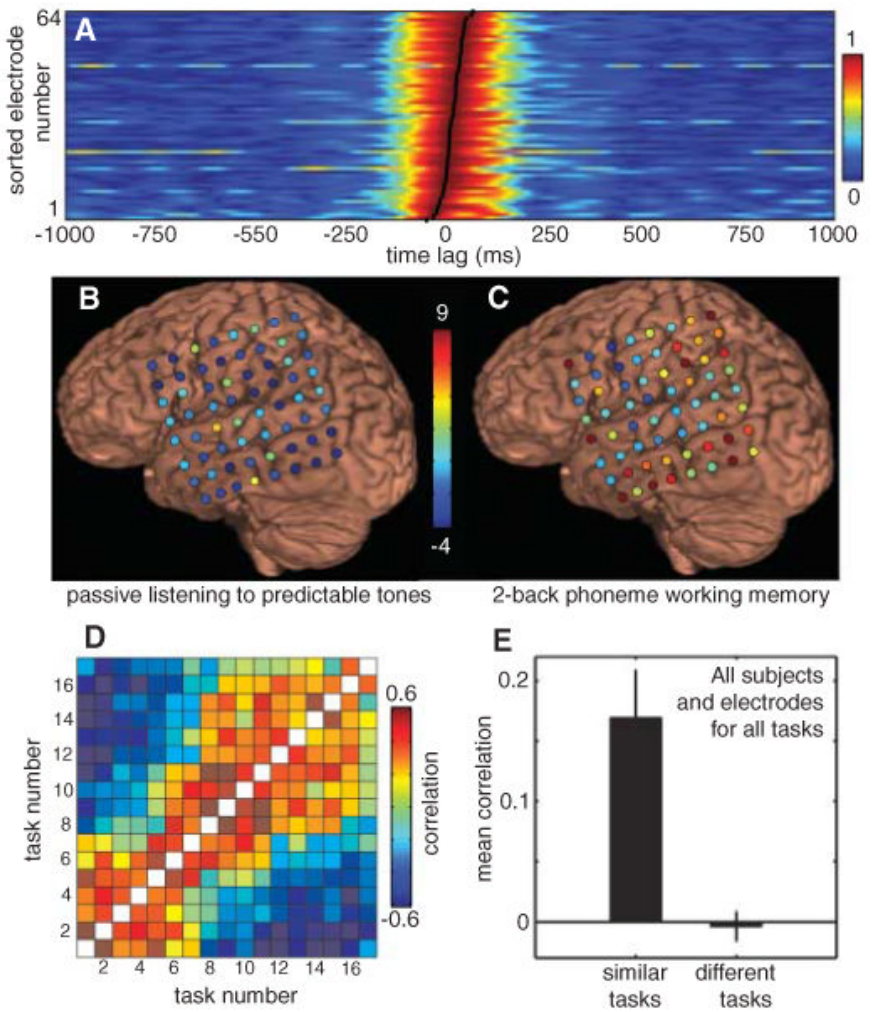

Fig. 3.

Task-specific changes in the spatial pattern of theta/HG coupling strength. (A) Theta/HG coupling strength falls to chance at large time lags. Modulation index (25)asa function of lag for all electrodes over all tasks from subject 5 . Electrodes are sorted by the time-lag $\tau_{\max }$ associated with maximal coupling (black line). For ease of comparison, horizontal traces were renormalized so that the peak value for each channel is one (see also fig. S8). (B) The change in modulation index values from the mean for all electrodes in subject 2 during one task (passive listening to predictable tones). (C) As in (B), for a difficult working memory task. Subjects listened to a list of phonemes and responded when the current phoneme and the phoneme presented two items earlier were identical. (D) Similar tasks evoke similar spatial patterns of theta/HG coupling. Correlation matrix for all tasks in subject 2. Tasks: 1 to 4, passive listening to tones or phonemes; 5 , mouth motor activation; 6 , verb generation; 7 , hand motor activation; 8 to 11 auditory working memory; 12 and 13, linguistic target detection; 14 to 17 , auditoryvibrotactile target detection (see SOM text). (E) Mean correlation and standard error between similar tasks (positive, $P<0.01$, corrected, 58 task pairs) as well as different tasks (not significant, 617 task pairs) for all electrodes in all subjects over all tasks. 